\title{
Protective effect of Bacillus Calmette Guerin (BCG) against leprosy: a population-based case- control study in Nagpur, India
}

\author{
SANJAY P. ZODPEY ${ }^{1, b}$, B. S. BANSOD ${ }^{2}$, \\ S. N. SHRIKHANDE ${ }^{3}$, B. R. MALDHURE ${ }^{4}$ AND \\ S. W. KULKARNI ${ }^{5}$ \\ ${ }^{1}$ Clinical Epidemiology Unit, and Dept of Preventive and Social \\ Medicine, Govt Medical College, Nagpur, MS, India \\ ${ }^{2}$ Assistant Directorate Health Services (Leprosy) Nagpur, Govt of \\ Maharashtra, India \\ ${ }^{3}$ Dept of Microbiology, Govt Medical College, Nagpur, MS, India \\ ${ }^{4}$ Dept of Medicine, Panjabrao Deshmukh Medical College, Amravati, \\ MS, India \\ ${ }^{5}$ Jawaharlal Nehru Medical College, Sawangi Meghe, Wardha, MS, \\ India
}

\section{Accepted for publication 1 June 1999}

Summary A population-based pair-matched case-control study was carried out in an urban community, Nagpur, India, to estimate the effectiveness of BCG vaccination in the prevention of leprosy. The study included 212 cases of leprosy (diagnosed by WHO criteria), below the age of 35 years, detected during a leprosy survey conducted by the Government of Maharashtra over a population of 20,03,325. Each case was pair-matched with one neighbourhood control for age, sex and socioeconomic status. A significant protective association between BCG and leprosy was observed $(\mathrm{OR}=0.40,95 \% \mathrm{CI}=0 \cdot 23-0 \cdot 68)$. The overall vaccine effectiveness (VE) was estimated to be $60 \%$ (95\% CI=32-77). The BCG effectiveness against multibacillary and paucibacillary leprosy was $72 \%(95 \% \mathrm{CI}=35-88)$ and $45 \%(95 \%$ $\mathrm{CI}=3-73$ ), respectively. Vaccine was more effective during the first decade of life, among females and in lower socioeconomic strata. The overall prevented fraction was $39 \%(95 \% \mathrm{CI}=16-58)$. In conclusion, this first ever population-based case control study performed in Central India, identified a beneficial role of BCG vaccination in prevention of leprosy in study population.

\section{Introduction}

Seven large controlled trials ${ }^{1-7}$ have been conducted to assess the protective effect of BCG vaccination against leprosy. The efficacy observed for the vaccine in these studies has varied

Correspondence to: Dr Sanjay P. Zodpey, 305, Hanuman Nagar, Nagpur 440 009, M.S., India 
from approximately $20 \%$ in Myanmar (Burma) ${ }^{3}$ to $80 \%$ in Uganda. ${ }^{6}$ During the last few years, the effectiveness of BCG was also studied by several case-control studies ${ }^{8-16}$ which provided consistent results with the prospective trials when considering all forms of leprosy (leprosy per se). However, there is a wide variation of efficacy and effectiveness reported from the different parts of the world. ${ }^{1-16}$ Although different results are reported in literature, findings of three studies ${ }^{4,714}$ added to the evidence that BCG vaccine affords greater protection against leprosy than against tuberculosis.

Of the total seven controlled trials ${ }^{1-7}$ and nine case control studies, ${ }^{8-16}$ evaluating role of BCG in prevention of leprosy carried out around the world, two trials, ${ }^{1,7}$ and one case control study ${ }^{13}$ are reported from India. The 1997 figures from WHO suggested that $70 \%$ of the world's leprosy patients are from India. With this background, the current case-control study was performed to estimate the effectiveness of BCG vaccination against leprosy in urban population of Nagpur city in Central India.

\section{Materials and methods}

\section{BACKGROUND ${ }^{17}$}

The Government of India started the National Tuberculosis Control Programme (NTCP) in 1962. The programme identified, districts as the working units where primary prevention, early detection, chemotherapy and case holding were the major activities. One of the primary prevention measures to which a lot of emphasis was given was BCG vaccination. During initial phase of the programme BCG was administered to a broader age range; however, since the implementation of the Expanded Programme of Immunization (EPI) in 1978 emphasis was given to BCG vaccination of infants. The BCG vaccine prepared from Danish 1331 strain in Guindy, Madras is used in a dose of $0.1 \mathrm{ml}$ intradermally ( $0.05 \mathrm{ml}$ dose for newborns). The vaccine is given without prior testing with purified protein derivatives.

\section{STUDY SETTING AND POPULATION}

The current population-based pair-matched case control study was carried out in urban population of Nagpur City, Maharashtra State, India. As a part of the National Leprosy Eradication Programme, a house to house cross sectional survey was performed by Government of Maharashtra over 20,03,325 urban population to detect hidden cases of leprosy and estimate overall prevalence, during January to March, 1998. This survey was conducted under direct supervision of Assistant Director Health Services (Leprosy) Govt of Maharashtra and involved undergraduate students from the local Medical College and urban leprosy workers. Provisional diagnosis done by surveyors was later cross checked and confirmed by leprologists. The list and detailed addresses of leprosy cases were then obtained from concerned authorities for the current study purpose.

Study investigators along with urban leprosy workers performed home visits to recruit cases and neighborhood controls. The study included 212 cases of leprosy (diagnosed by WHO criteria). ${ }^{18}$ As suggested by WHO, ${ }^{18}$ an individual was regarded having leprosy if he or she showed one of the following cardinal signs: hypopigmented or reddish skin lesion(s) with definite loss of sensation; damage to the peripheral nerves, as demonstrated by loss of sensation and weakness of the muscles of hands, feet or face; positive skin smears. When skin smears were not available or not dependable, more than five skin lesions were classified as 
multibacillary and skin lesions up to five were considered as paucibacillary leprosy. When skin smears were available and dependable, smear negative was considered as paucibacillary and smear positive as multibacillary leprosy. Nerve damage involving only one nerve trunk was considered as paucibacillary and involvement of many nerve trunks was considered as multibacillary leprosy. Diagnosis and classification was performed by leprologists. All the study subjects (including the cases) were below the age of 35 years, to take into account those born since the beginning of the NTCP in 1962. Each case was pair-matched with one neighbourhood control for age (within 1 year of the age of the case), sex and socioeconomic status (SES). For selection of a control, the immediate neighbourhood of a case was contacted and age, sex and SES was recorded. If the chosen neighbourhood could not be matched, the subject was excluded and the same procedure repeated for the next neighbourhood. Socioeconomic status was recorded using the modified Kuppuswamy scale ${ }^{19}$ of socioeconomic status classification, using occupation, education and per capita income as parameters. This is a 5-point scale, with class I representing the highest socioeconomic status (upper) and class V representing the lowest (lower). Class II, III and IV are represented by upper middle, lower middle and upper lower socioeconomic status, respectively.

\section{MEASUREMENT OF EXPOSURE TO BCG}

Evidence of BCG vaccination was determined by direct observation of a BCG scar at insertion of deltoid; immunization records if available and information from study subjects or parents in case of children. Cases or controls with missing data about BCG vaccination were excluded from the study. The measurement of exposure was thus carried out as per the guidelines given by Smith. ${ }^{20}$

\section{STATISTICAL ANALYSIS}

Odds ratios for the matched design were calculated as described by Greenberg and Ibrahim. ${ }^{21}$ The method described by Schlesselman ${ }^{22}$ was used for calculating $95 \%$ confidence intervals for the odds ratios. McNemar's $\chi^{2}$ test was also used. Subgroup analysis for matching variables was carried out separately. The effectiveness of BCG vaccination was calculated by the formula $(1-\mathrm{OR}) \times 100 \%$ where OR is the estimated odds ratio. The proportion of potential new cases that were prevented, the 'Prevented Fraction' was determined according to the method of Miettinen. ${ }^{23}$ The statistical analysis was done by using the MINITAB statistical package and dedicated Turbo $\mathrm{C}$ routines.

\section{Results}

A total of 400 cases (including active old cases) were recognized in leprosy survey. Prevalence of leprosy was estimated to be 2 per 10,000 in Nagpur urban population. Of these, 215 cases were aged less than 35 years. Three cases, who had missing records or uncertain about BCG vaccination, were not included. Hence the current study could include 212 cases of leprosy. Table 1 describes the subjects by the study characteristics. The majority of the cases were males aged 10-20 years and from the upper lower and lower middle classes of Kupuswamy's socioeconomic status scale. Because of small number of study subjects in other strata, classes I, II and III are merged to form one group and classes IV and V formed 
Table 1. Distribution of subjects by study characteristics

\begin{tabular}{lrr}
\hline & \multicolumn{1}{c}{ Cases } \\
Factors & $n=212(\%)$ & \multicolumn{1}{c}{$\begin{array}{c}\text { Controls } \\
n=212(\%)\end{array}$} \\
\hline Age (years) & & \\
$\quad<10$ & $34(16 \cdot 04)$ & $34(16.04)$ \\
$10-20$ & $114(53.77)$ & $114(53.77)$ \\
$>20$ & $64(30 \cdot 19)$ & $64(30 \cdot 19)$ \\
Sex & & \\
$\quad$ Male & $124(58 \cdot 49)$ & $124(58.49)$ \\
Female & $88(41.51)$ & $88(41 \cdot 51)$ \\
Socioeconomic status & & \\
$\quad$ Upper & $12(05.66)$ & $12(05.66)$ \\
$\quad$ Upper middle & $27(12.74)$ & $27(12.74)$ \\
$\quad$ Lower middle & $76(35.85)$ & $76(35.85)$ \\
$\quad$ Upper lower & $92(43.40)$ & $92(43.40)$ \\
$\quad$ Lower & $5(02.35)$ & $5(02.35)$ \\
Exposure to BCG & $92(43.40)$ & $120(56.60)$ \\
& & \\
\hline
\end{tabular}

another group. The prevalence of exposure was $43.40 \%$ and $56.60 \%$ in cases and controls respectively. The study included $135(63.68 \%)$ cases of paucibacillary leprosy and 77 (36.32\%) cases of multibacillary leprosy.

Table 2 describes subgroup analysis of paired data. The significant protective association $(\mathrm{OR}=0 \cdot 40,95 \% \mathrm{CI}=0 \cdot 24-0 \cdot 69)$ between $\mathrm{BCG}$ and leprosy is seen from this table. The subgroup analysis revealed that the odds ratios were lower in the less than 10 years age group and in females, but these were not significantly different from the other groups. The different socioeconomic strata also did not differ significantly from each other with respect to the estimated odds ratios. The estimates of odds ratios were lower for multibacillary leprosy as compared to paucibacillary leprosy, but this difference too was not statistically significant. The vaccine effectiveness and prevented fraction were maximum for females, multibacillary

Table 2. Subgroup analysis of paired data

\begin{tabular}{|c|c|c|c|c|}
\hline Groups & Odds ratio & $95 \% \mathrm{CI}$ & $\begin{array}{l}\text { McNemar's } \\
\text { Chi-square }\end{array}$ & $P$ value \\
\hline All pairs & 0.40 & $0 \cdot 24-0.69$ & $11 \cdot 87$ & $<0.001$ \\
\hline \multicolumn{5}{|l|}{ Type of leprosy } \\
\hline Multibacillary & $0 \cdot 28$ & $0 \cdot 12-0 \cdot 65$ & $10 \cdot 12$ & $<0.001$ \\
\hline Paucibacillary & 0.55 & $0 \cdot 27-0.97$ & 2.94 & $>0.05$ \\
\hline \multicolumn{5}{|l|}{ Age (years) } \\
\hline$<10$ & $0 \cdot 20$ & $0.04-0.91$ & $5 \cdot 33$ & $<0.05$ \\
\hline $10-20$ & 0.41 & $0 \cdot 20-0 \cdot 86$ & 5.76 & $<0.05$ \\
\hline$>20$ & $0 \cdot 54$ & $0 \cdot 22-1 \cdot 35$ & $1 \cdot 80$ & $>0.05$ \\
\hline \multicolumn{5}{|l|}{ Sex } \\
\hline Male & $0 \cdot 50$ & $0 \cdot 25-0.99$ & 4.00 & $<0.05$ \\
\hline Female & $0 \cdot 30$ & $0 \cdot 13-0 \cdot 71$ & $8 \cdot 53$ & $<0.01$ \\
\hline \multicolumn{5}{|l|}{ Socioeconomic status } \\
\hline $\begin{array}{l}\text { Upper middle \& } \\
\text { lower middle }\end{array}$ & 0.44 & $0 \cdot 22-0 \cdot 89$ & $5 \cdot 44$ & $<0.05$ \\
\hline Upper lower \& lower & $0 \cdot 36$ & $0 \cdot 16-0 \cdot 82$ & $6 \cdot 53$ & $<0.01$ \\
\hline
\end{tabular}


Table 3. Estimates of BCG effectiveness and prevented fraction calculated from odds ratios

\begin{tabular}{|c|c|c|c|c|}
\hline \multirow[b]{2}{*}{ Groups } & \multicolumn{2}{|c|}{ BCG effectiveness } & \multicolumn{2}{|c|}{ Prevented fraction } \\
\hline & $\%$ & $95 \% \mathrm{CI}$ & $\%$ & $95 \% \mathrm{CI}$ \\
\hline All pairs & 60 & $31-76$ & 39 & $16-58$ \\
\hline \multicolumn{5}{|l|}{ Type of leprosy } \\
\hline Multibacillary & 72 & $35-88$ & 38 & $11-63$ \\
\hline Paucibacillary & 45 & $3-73$ & 31 & $2-60$ \\
\hline \multicolumn{5}{|l|}{ Age (years) } \\
\hline$<10$ & 80 & $9-96$ & 60 & $4-81$ \\
\hline $10-20$ & 59 & $14-80$ & 38 & $7-64$ \\
\hline$>20$ & 46 & $-35-79$ & 29 & $-14-63$ \\
\hline \multicolumn{5}{|l|}{ Sex } \\
\hline Male & 50 & $1-75$ & 30 & $1-56$ \\
\hline Female & 70 & $29-87$ & 51 & $16-75$ \\
\hline \multicolumn{5}{|l|}{ Socioeconomic status } \\
\hline $\begin{array}{l}\text { Upper middle \& } \\
\text { lower middle }\end{array}$ & 56 & $11-78$ & 36 & $5-62$ \\
\hline Upper lower \& lower & 64 & $18-84$ & 42 & $9-69$ \\
\hline
\end{tabular}

leprosy, lower socioeconomic group and subjects in the age group of less than 10 years (Table 3). The BCG effectiveness and prevented fraction for whole study group were calculated to be $60 \%(31-76)$ and $39 \%(16-58)$, respectively.

\section{Discussion}

The rationale for the use of $\mathrm{BCG}$ for prevention of leprosy was based on the postulate by Fernande ${ }^{24}$ of a protective effect of such vaccination. This postulate was supported by the observation that when household controls of leprosy patients were vaccinated repeatedly with BCG, the Mitsuda reaction became positive. ${ }^{25}$ This observation was later confirmed by several workers and led to a series of studies to evaluate the protective efficacy of BCG against leprosy. ${ }^{26}$ So far, seven controlled trials ${ }^{1-7}$ have been conducted to assess the role of BCG in prevention of leprosy, which included two trials from India. ${ }^{1,7}$ Since Smith ${ }^{20}$ recommended the use of case-control design to evaluate the effectiveness of BCG against tuberculosis, investigators shif ted their choice of design to case control studies. ${ }^{8-16}$ Although this design has obvious advantages (quicker, cheaper, free from ethical considerations), it has a major weakness: susceptible for biases. ${ }^{21}$ The prospective studies are supposed to provide results without bias. The case-control study may not have control over these inherent biases. This methodological issue has also been attributed to the differences in the vaccine estimates measured by different study designs. This may be the reason why the results differ in a casecontrol study and a prospective study in South India in two adjacent districts. In spite of the well understood limitations of case-control design, it has been widely used and so far nine case-control studies of BCG vaccination and leprosy are reported world wide. Age, sex, socioeconomic parameters and area of residence have conventionally been considered as the confounding factors. Most of the case control studies have therefore used these as matching factors. The current study also have used these variables as matching factors. Selection of 
neighbourhood controls and use of socioeconomic status as a matching variable appears to have created a good balance between cases and controls with respect to many of the socioeconomic parameters which could have had a bearing on the chance of receiving $\mathrm{BCG}$, on the one hand, or the risk of disease detection on the other.

The crude criteria of exposure classification have been used in this study, which could have resulted in misclassification to a certain extent. However, this bias is quite small if $90 \%$ of vaccinations leave scars and around $70 \%$ of the population is vaccinated. ${ }^{20}$ Nevertheless, this possibility is unlikely to alter the results of this study. However, it cannot be overlooked that the reported sensitivity of scar reading (the proportion of vaccinated individuals who develop a recognizable scar) has been reported to vary from $98.9 \%$ in South India, as assessed 4 years afterwards, to $60 \%$ among Swedish children 14 years after having been vaccinated at birth. ${ }^{27}$ Moreover, the specificity of scar reading (the proportion of individuals who have no evidence of a BCG-like scar among those who have never been vaccinated) is more problematic to measure, since it is difficult to confirm a negative vaccination history. ${ }^{27}$ Additionally, selective susceptibility in individuals in whom the BCG scars disappeared for leprosy particularly of the MB form cannot be ruled out. In this case, higher estimates of vaccine effectiveness for MB leprosy can be attributed to this phenomenon. In this respect, vaccine efficacy studies based upon scar evidence of vaccination should consider the effect of this bias on vaccine efficacy. In some circumstances, it may be possible to validate scar information against documentary evidence of vaccination.

The present study demonstrated $27 \%$ statistically non-significant excess protective effectiveness of BCG against multibacillary leprosy as compared to paucibacillary leprosy. A few earlier studies ${ }^{10,11}$ have reported higher estimates of BCG effectiveness against multibacillary leprosy as compared to paucibacillary leprosy, which has significant public health implications because multibacillary disease is thought to be a major source for the spread of $M$. leprae in the community. Thus in general the findings of the present study are consistent with the theory that BCG vaccination brings about a shift in the immune response to a higher level of cell mediated immunity, and thereby offers protection especially against the more severe multibacillary form of the disease. ${ }^{10,13}$ When this phenomenon is operative, case control studies on the protective effect of BCG vaccination on paucibacillary leprosy suggest that there is no or less effect when, in fact, there is an important effect; BCG shifting of the potential multibacillary patients in the direction of the clinically less severe paucibacillary form.

The present study recognized that the protective effect of BCG vaccination in the age group less than 10 years was higher than in the 10-20 years and more than 20 years age groups. The policy of BCG immunization during infancy is followed in India. Hence, the age of a vaccinated individual approximates the years since vaccination. It is logical to believe that as the time since vaccination advances, immunity decreases. Hence, this may be the reason for the decline in effectiveness of BCG as the age advances in the present study. Earlier studies have indicated that the protective efficacy of BCG vaccine increases with the interval of time since vaccination. ${ }^{14-16}$ However, another study from India ${ }^{13}$ observed that with advancing age, BCG effectiveness declines in females. The current study demonstrated $20 \%$ excess protective effectiveness of BCG vaccination in females as compared to males. This finding is in agreement with earlier reports. ${ }^{12-14}$ The only case control study carried out in India, ${ }^{13}$ investigating the relationship between leprosy and BCG, has also reported higher estimates of BCG vaccination in females. However, few case referent studies ${ }^{11-16}$ have reported an excess protective effectiveness of BCG vaccination in 
males. Although vaccine effectiveness in this study was $8 \%$ higher in upper lower and lower socioeconomic strata, it was non-significant and could be because of chance.

The present study showed 60\% (31-76) vaccine effectiveness and 39\% (16-58) prevented fraction with the use of BCG vaccination in the prevention of leprosy. This finding is in agreement with earlier studies, ${ }^{8,10,14,15}$ which evaluated the role of BCG in prevention of leprosy in other parts of the world. Earlier controlled trials ${ }^{1-7}$ and case control studies ${ }^{8-16}$ have demonstrated the efficacy and effectiveness of BCG in the range of $20-81 \%$. The estimate of vaccine effectiveness $(60 \%)$ observed in this study is relatively closer to the upper estimate of this range. Moreover the proportion of potential new cases that were prevented, the 'prevented fraction' was calculated to be $39 \%$ (16-58), i.e. the vaccination programme prevented 39 of every 100 cases that would have occurred in its absence. The findings of this study thus indicated that BCG vaccination was effective against leprosy in Central India. This finding is encouraging for prevention of a disease that has been more prevalent in this country as compared to other parts of the world. However, an earlier case control study performed in this country, in South India, ${ }^{13}$ has reported relatively low estimates of BCG vaccine effectiveness in the prevention of leprosy. Hence, with this background and fortified by the fact that vaccine efficacy/effectiveness seems to be dependent more on geography and environmental factors than on vaccine strain, further studies should be carried out to evaluate the role of BCG in prevention of leprosy in different parts of the country.

Conventionally, BCG vaccination is practised worldwide for prevention of tuberculosis. Its effectiveness against leprosy is the additional advantage. Even though it is moderately effective against both these mycobacterial diseases, it has a beneficial role, particularly in countries like India, where both these diseases are widely prevalent. As observed in the current study, its role in prevention of MB leprosy as compared to PB leprosy has definite public health implications. Additionally, the findings of the present study, that, as the age advances BCG effectiveness declines, which is supported by other studies also, call for a public health recommendation on thinking of a booster dose of BCG at the appropriate age.

\section{References}

${ }^{1}$ Chaudhury S, Hazra SK, Saha B, Mazumder B, Biswas PC, Chattopadhya D, Saha K. A eight year field trial on antileprosy vaccines among high risk household contacts in the Calcutta Metropolis. Int J Lepr, 1993; 62: 389-394.

2 Convit J, Smith PG, Zuniga M, Sampson C, Ulrich M, Plata JA, Silva J, Molina J, Salgado A. A immunoprophylactic trial with combined Mycobacterium leprae/BCG vaccine against leprosy: preliminary results. Lancet, 1992; 339: 446-450.

3 Lwin K, Sundaresan T, Gyi MM, Bechelli LM, Tamondong C, Garbajosa PG, Sansarricq H, Noordeen SK. BCG vaccination of children against leprosy: fourteen year findings of the trial in Barma. Bull WHO, 1985; 63: 1069-1078.

4 Ponnighaus JM, Fine PEM, Sterne JAC, Wilson RS, Msosa E, Gruer PJK, Jenkins PA, Lucas SB, Liomba G, Bliss L. Efficacy of BCG against leprosy and tuberculosis in northern Malawi. Lancet, 1992; 339: 636-639.

5 Scott GC, Russell DA, Boughton CR, Vincin DR. Untreated leprosy. Probability of shifts in Ridley-Jopling classification. Development of 'flares' or disappearance of clinically apparent disease. Int J Lepr, 1978; 44: $110-122$

6 Stanley SJ, Howland C, Stone MM, Sutherland I. BCG vaccination of children against leprosy in Uganda. Final results. J Hyg Camb, 1981; 87: 233-248.

7 Tripathy SP. The case for BCG. Ann Natl Acad Med Sci (India), 1983; 19: 12-21.

8 Abel L, Cua VV, Oberti J, Lap VD, Due IK, Grossi J, Lagrange PH. Leprosy and BCG in Southern Vietnam (letter). Lancet, 1990; 335: 1536.

9 Baker DM, Nguyen Van Tam JS, Smith SJ. Protective efficacy of BCG vaccine against leprosy in Southern Malawi. Epidemiol Infect, 1993; 111: 21-25. 
${ }^{10}$ Boelens JJ, Kroes R, Beers SV, Lever P. Protective effect of BCG against leprosy in South Sulawesi, Indonesia. Int J Lepr, 1995; 63: 456-457.

11 Convit J, Smith PG, Zuniga M, Sampson C, Ulrich M, Plata JA, Silva J, Molina J, Salgado A. BCG vaccination protects against leprosy in Venezuela: a case-control study. Int J Lepr, 1993; 61: 185-191.

12 Fine PEM, Ponnighaus JM, Maine N, Clarkson JA, Bliss L. The protective efficacy of BCG against leprosy in northern Malawi. Lancet, 1986; ii: 499-502.

13 Muliyil J, Nelson KE, Diamond EL. Effect BCG on the risk of leprosy in an endemic area: a case-control study. Int J Lepr, 1991; 59: 229-236.

14 Orege PA, Fine PEM, Lucas SB, Obura M, Okela C, Okuku P. Case-control study of BCG vaccination as a risk factor for leprosy and tuberculosis in Western Kenya. Int J Lepr, 1993; 61: 542-549.

15 Rodrigues MLO, Silva SA, Neto JCA, De Andrade Alss, Martelli CMT, Zicker F. Protective effect of intradermal BCG against leprosy: a case-control study in Central Brazil. Int J Lepr, 1992; 60: 335-339.

16 Thuc NV, Abel L, Lap VD, Oberti J, Lagrange PH. Protective effect of BCG against leprosy and its subtypes: a case-control study in Southern Vietnam. Int J Lepr, 1994; 62: 532-538.

17 Suri AK. National Programme for control of tuberculosis. National Institute of Health and Family Welfare, New Delhi, 1988; pp 11-34.

18 WHO. A guide to eliminating leprosy as a public health problem. Geneva: WHO/LEP/95.1, 1995; pp 15-26.

19 Mahajan BK, Gupta MC. Social environment. In: Textbook of preventive and social medicine. New Delhi: Jaypee Bros, 1991; pp 82-86.

${ }^{20}$ Smith PG. Retrospective assessment of the effectiveness of BCG vaccination against tuberculosis using the case-control method. Tubercle, 1982; 62: 23-35.

21 Greenberg RS, Ibrahim MA. The case-control study. In: Holland WW, Detels R, Knox G, eds. Oxford textbook of public health. London: Oxford University Press, 1985; pp 123-143.

22 Schlesselman JJ. Case-control studies: design, conduct, analysis. New York: Oxford University Press, 1982; pp 171-226.

23 Miettinen OS. Proportion of disease caused or prevented by a given exposure trait or intervention. Am J Epidemiol, 1974; 99: 325-332.

${ }^{24}$ Fernandez JM. The early reaction induced by lepromin. Int J Lepr, 1940; 8: 1-14.

${ }^{25}$ Convit J, Rassi E. Lepromin and tuberculin tests in Venezuelan leprosy foci: induction of lepromin reaction by BCG vaccination. Int J Lepr, 1954; 22: 303-310.

${ }^{26}$ Fine PEM. BCG vaccination against tuberculosis and leprosy. Br Med Bull, 1988; 44: 691-703.

27 Fine PEM, Ponnighaus JM, Maine N. The distribution and implications of BCG scars in northern Malawi. Bull WHO, 1989; 67: 35-42. 